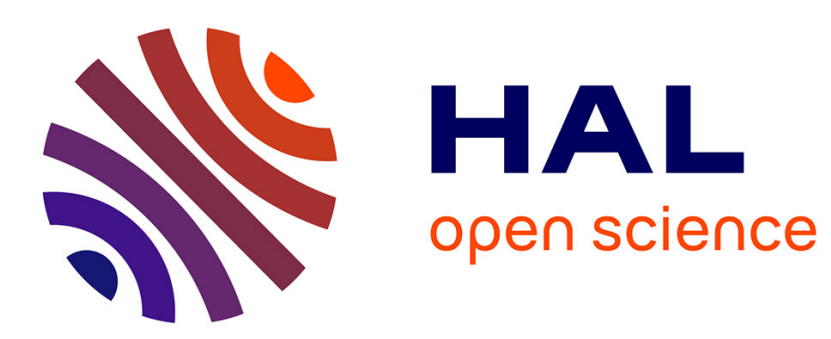

\title{
Stochastic digital holography for visualizing inside strongly refracting transparent objects
}

\author{
Jean-Michel Desse, Pascal Picart
}

\section{To cite this version:}

Jean-Michel Desse, Pascal Picart. Stochastic digital holography for visualizing inside strongly refracting transparent objects. Applied optics, 2015, 54 (1), pp.A1-A8. 10.1364/AO.54.0000A1 . hal01082855

\section{HAL Id: hal-01082855 \\ https://hal.science/hal-01082855}

Submitted on 14 Nov 2014

HAL is a multi-disciplinary open access archive for the deposit and dissemination of scientific research documents, whether they are published or not. The documents may come from teaching and research institutions in France or abroad, or from public or private research centers.
L'archive ouverte pluridisciplinaire HAL, est destinée au dépôt et à la diffusion de documents scientifiques de niveau recherche, publiés ou non, émanant des établissements d'enseignement et de recherche français ou étrangers, des laboratoires publics ou privés. 


\title{
Stochastic digital holography for visualizing inside strongly refracting transparent objects
}

\author{
Jean-Michel Desse ${ }^{1, *}$ and Pascal Picart ${ }^{2,3}$ \\ 'ONERA-The French Aerospace Lab, 5 Boulevard Paul Painlevé, 59000 LILLE, France \\ ${ }^{2}$ LUNAM Université, Université du Maine, CNRS UMR 6613, LAUM, Avenue Olivier Messiaen, \\ 72085 LE MANS Cedex 9, France \\ 33École Nationale Supérieure d'Ingénieurs du Mans, rue Aristote, 72085 LE MANS Cedex 9, France \\ *Corresponding author: Jean-Michel.Desse@onera.fr
}

Received 15 September 2014; accepted 6 October 2014;

posted 13 October 2014 (Doc. ID 223085); published 6 November 2014

\begin{abstract}
This paper presents a digital holographic method to visualize and measure refractive index variations, convection currents, or thermal gradients, occurring inside a transparent and refracting object. The proof of principle is provided through the visualization of refractive index variation inside a lighting bulb. Comparison with transmission and reflection holography is also provided. A very good agreement is obtained, thus validating the proposed approach. (C) 2014 Optical Society of America

OCIS codes: (090.1995) Digital holography; (090.2880) Holographic interferometry; (090.5694)

Real-time holography; (120.5050) Phase measurement.

http://dx.doi.org/10.1364/AO.54.0000A1
\end{abstract}

\section{Introduction}

Digital holography has become properly available since its confirmation was established in 1994 [1-3]. For a few years, digital holography has become a very stimulating topic for the scientific community working in this area. Spectacular applications have been demonstrated, such as microscopy (also known as quantitative phase imaging or interferometric phase microscopy) [4-14], material properties [15], surface shape [16], polarization imaging [17], displacement field measurements [18,19], or vibrations [20-23]. In addition, an increasing number of applications rely on the possibilities of using digital color holography to record and reconstruct colored objects at high precisions by using a simple optical setup [24-41]. The main motivation for digital holography is related to the possibility of recording the optical phase of the scene. Instead of classical photography which is only recording the square of the amplitude of the complex

$1559-128 \mathrm{X} / 15 / 0100 \mathrm{~A} 1-08 \$ 15.00 / 0$

(C) 2015 Optical Society of America optical field, digital holography provides full information. This unconventional imaging method offers opportunities for studying a large panel of physical events: study of internal field grows in lithium niobate crystal [42], study of the clarinet reed behavior in the auto-oscillation regime [43], imaging nanoparticles in living cell environments [44], visualizing fish embryo [45], or visualizing through smoke with infrared digital holography [46]. Digital holography is also a powerful approach for investigating fluids and to visualize/measure dynamic flows [29,47-49]. However, visualizing flows inside a transparent but strongly refracting object remains a challenge for digital holography. To illustrate this purpose, consider the three main modes for recording a digital hologram: object having a rough surface (opaque object), pure phase object, and strongly refracting transparent object. Figure 1 shows these three situations. Figure 1(a) shows the case of the rough object: the object wave front is speckled, and the recorded hologram also includes speckle grains modulating the fringe pattern. In Fig. 1(b), the phase object produces a relatively smooth wave front, leading to 

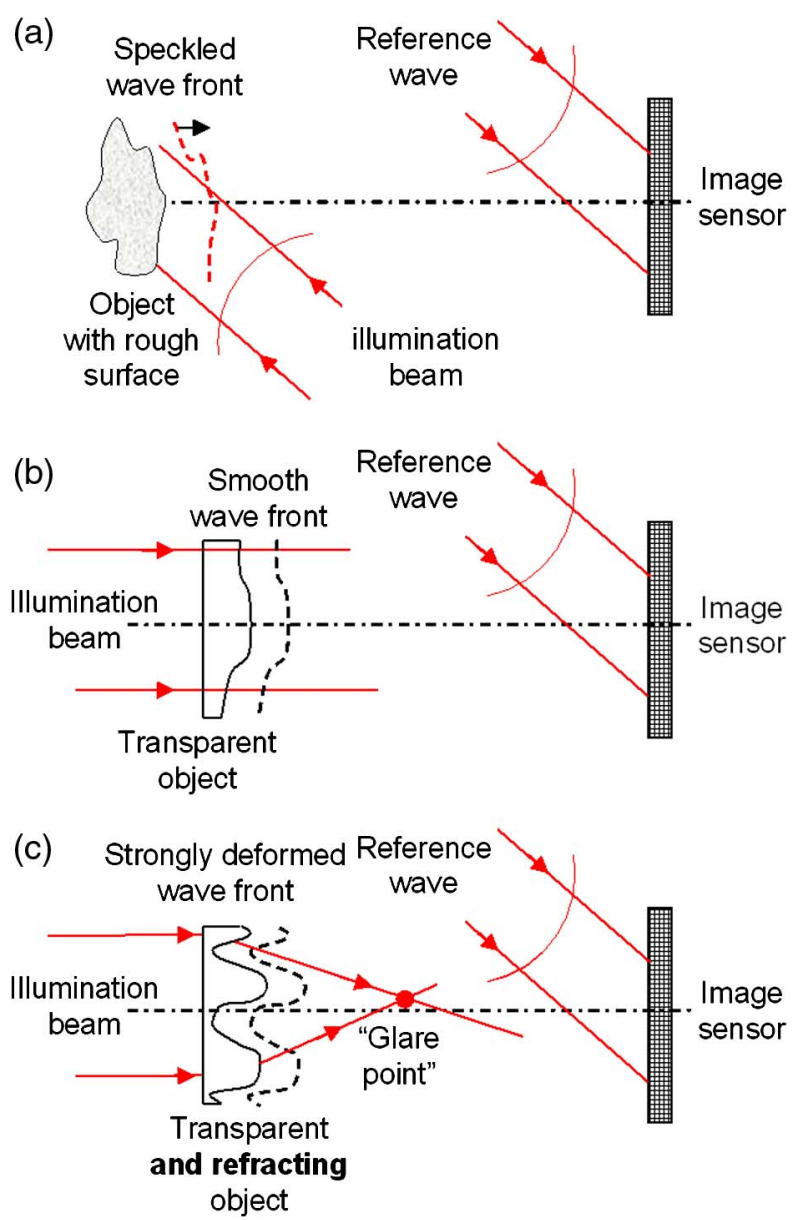

Fig. 1. Scheme for recording objects having different optical properties, (a) rough object, (b) pure phase object, and (c) transparent and strongly refracting object.

microfringes without strong speckle modulation. In Fig. 1(c), the object is transparent but also strongly refracting light: the object wave is then strongly deformed after passing through the medium, and generates glare points between the object and sensor planes.

Figure 2 shows the recorded holograms and reconstructed images obtained from the three different recording schemes and object types. Figure 2(a) shows on the left the speckled digital hologram and on the right the full field image reconstructed using the discrete Fresnel transform; one can easily see an in focus $1 €$ coin localized in the upper left corner of the field of view. In Fig. 2(b), the left part shows a three color digital hologram in which one can recognize the microfringes. After digital processing, the optical path difference can be measured (shown on the right) [47]. Figure 2(c) illustrates the case of the strongly refracting object constituted by a low quality plastic tank filled with water and including small balls; the digital hologram is unstructured. Using the discrete Fresnel transform, the reconstructed amplitude image (provided on the right) does not include any pertinent image of the tank and balls. The glare points due to the strong refractions (secondary (a)

(b)
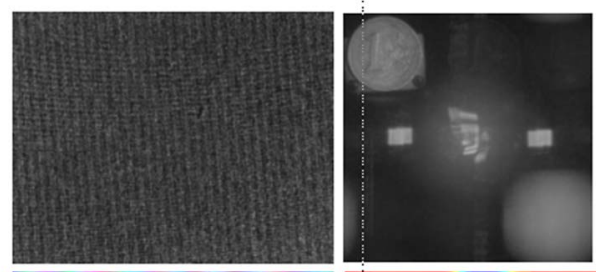

(c)
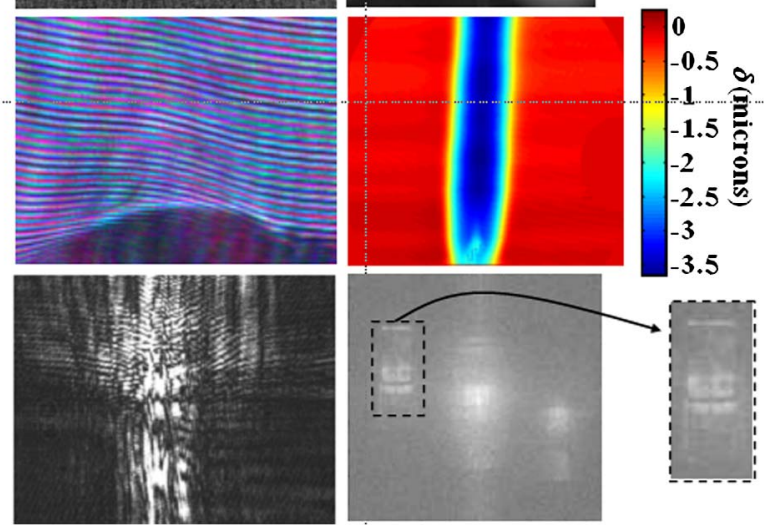

Fig. 2. Three digital holograms and reconstructions, (a) rough object, (b) pure phase object, and (c) transparent and strongly refracting object.

focal points) are numerically back propagated, thus inducing blur of the image.

Other objects, such as a glass ball, a bulb, a glass container, a glass flask, ..., are not opaque but they are refracting light and measuring inside is not straightforward. Then, observing physical phenomena, such as refractive index variations, convection currents, or thermal gradients, occurring inside the object require specific methods. The approaches proposed in the past $[29,47-54]$ are very powerful to measure flows or temperature fields and very appropriated when the envelope including the gas/liquid is relatively smooth and transparent (i.e., not strongly refracting).

In digital holography, the use of a stochastic screen to analyze flows was first discussed by Demoli et al. [29]. They used a three-color Fourier holography setup combined with subtraction holography [55], and a ground glass was adjusted in front of the object, between its plane and the sensor plane. The test object was a transparent tank filled with oil and heated to create convective thermal flows which lead to refractive index variations in the oil. The object to be recorded (i.e., the oil and its index variations) being a pure phase type, the ground glass plate was used to diffusely scatter the object beam. Experimental results show qualitative color movie from the sequence of RGB reconstructed amplitude images. In that case, the ground glass plate acts as a screen on which are projected the Newton fringes from the interferences in the tank. Although the results are quite impressive, no quantitative measurement of convection in the fluid was demonstrated.

In this paper, we would like to take benefit of stochastic holography to overcome the problems induced by strongly refracting objects, in order to be 
able to exhibit the phase changes inside the object without suffering from any image distortion. This paper is organized as follows; Section 2 describes the proposed method, optimization parameters and reconstruction process; Section 3 provides a proof of principle by considering the case of a lightening bulb which is submitted to a current to produce light; Section 4 proposes a comparison with silver-halide real-time holographic interferometry; and Section 5 gives conclusions and few perspectives to this study.

\section{Stochastic Digital Holography}

\section{A. Experimental Setup}

The approach that can be quite adapted to visualize inside a strongly refracting object is described in Fig. 3. A cw laser at $\lambda=532 \mathrm{~nm}$ is split into a reference and an object wave. The reference wave is propagating to the sensor area by using a 50\% cube. It is beforehand spatially filtered and expanded to produce a plane and smooth wave front. The cube is slightly tilted to produce an off-axis hologram. The object wave is expanded and is impacting a ground glass localized behind the object under interest. The test object is a transparent and refracting specimen and is physically localized at distance $d_{0}$ from the sensor plane. Due to the stochastic illumination, a speckle wave is produced by the ground glass. After passing through the specimen, this speckle wave is strongly deformed but does not lose its speckle nature. This means that the digital hologram produced by the coherent mixing between the reference and the object wave includes speckle and looks like that of Fig. 2(a) (left). The sensor includes $N \times M=1920 \times 1440$ pixels with pitches $p_{x}=p_{y}=$ $3.65 \mu \mathrm{m}$, with conventional frame rate.

\section{B. Recording Large Objects}

The Shannon conditions impose a general rule [56]: the reconstructed object must be included in the reconstructed field. In the Fresnel approach, with reconstruction distance $d_{r}$, the width of the reconstructed field is equal to $\lambda d_{r} / p_{x}$. So, the dimension of the experimental setup is directly proportional to the size of the object. For example, using a wavelength at $532 \mathrm{~nm}$, to study an object sized $15 \mathrm{~cm} \times 15 \mathrm{~cm}$, with pixels $3.65 \mu \mathrm{m}$, the distance must be at least $4.1 \mathrm{~m}$ [56]. In order to satisfy the Shannon criteria with an acceptable size of the setup, and to maintain an acceptable illumination (a $5 \mathrm{~m}$

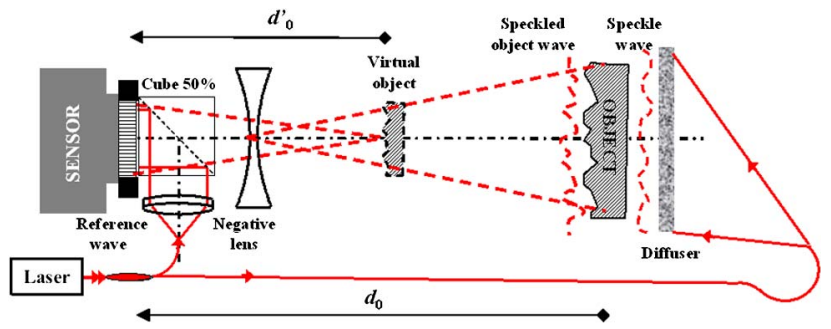

Fig. 3. Experimental setup for stochastic digital holography. long setup will require high laser power), the use of a negative lens was introduced by Schnars et al. [57], then generalized to a lens assembly by Mundt and Kreis [58]. The negative optical system provides a virtual image of the object at the closest distance from the sensor. This virtual object is reduced (optical magnification $\left|\gamma_{\text {opt }}\right|<1$ ) and in the same direction as the physical object. In Fig. 3 , the negative lens placed just before the combining cube in front of the sensor produces a virtual image localized at distance $d_{0}^{\prime}$ from the sensor. The digital hologram is then produced with the speckled virtual image wave. This leads to a more compact system compared to the case where the lens is not used.

The optimization of the off-axis setup has to satisfy the basic rules about the Shannon conditions [56]. Particularly, the focal length of the lens has to be judiciously calculated. We choose as criterion that the observation angle from the sensor to the virtual image has to fulfill this condition:

$$
\theta_{\max }=\frac{\lambda}{(4-2 \alpha) \max \left(p_{x}, p_{y}\right)},
$$

where $\alpha=\Delta a / a$ is the accepted tolerance in the superposition of the useful +1 order and the 0 order, $a$ being the width of the object. Figure 4 illustrates this rule for a circular [Fig. 4(a)] and a square [Fig. 4(b)] object.

\section{Holographic Recording and Reconstruction}

In the recording plane, the hologram is expressed as [1]:

$$
H=|R|^{2}+\left|O^{\prime}\right|^{2}+R^{*} O^{\prime}+R O^{*},
$$

where $R\left(x^{\prime}, y^{\prime}\right)=a_{R} \exp \left[-2 i \pi\left(u_{0} x^{\prime}+v_{0} y^{\prime}\right)\right](i=\sqrt{ }-1)$ is the reference plane wave, with spatial frequency $\left\{u_{0}, v_{0}\right\}$ related to the tilt provide by the cube, and $O^{\prime}$ is virtual object wave. The object wave at the recording plane is given by a convolution formula (* means convolution):

$$
O^{\prime}\left(x, y, d_{0}\right)=\left(A^{\prime} * h\right)(x, y),
$$

where $A^{\prime}(x, y)=A_{0}(x, y) \exp \left[i \psi_{0}(x, y)\right]$ is the virtual object wave front at the virtual object plane, and $h\left(x, y, d_{0}\right)$ is the impulse response of free space

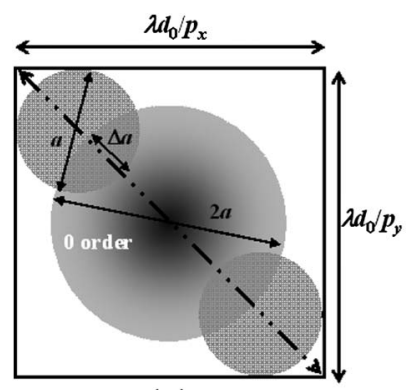

(a)

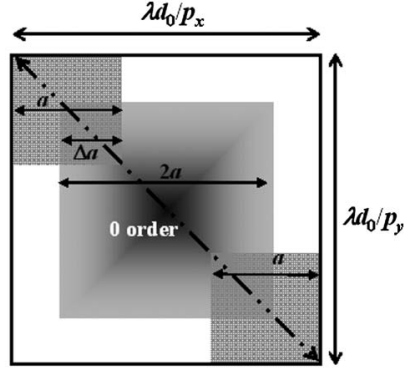

(b)
Fig. 4. Optimization rule for (a) circular object and (b) a square object. 
propagation along distance $d_{0}^{\prime}$. According to Ref. [59], we have:

$$
h\left(x, y, d_{0}\right)=-\frac{i d_{0}^{\prime}}{\lambda} \frac{\exp \left\lfloor 2 i \pi / \lambda \sqrt{d_{0}^{\prime 2}+x^{2}+y^{2}}\right\rfloor}{d_{0}^{\prime 2}+x^{2}+y^{2}} .
$$

There are primarily two ways to compute the reconstruction of digitally recorded holograms. These methods are based on Eq. (3) and its possible different interpretations [60]. At any reconstruction distance $d_{r}$, which may be different from $d_{0}^{\prime}$, the numerically reconstructed hologram corresponds to the discrete version of the equation (discrete Fresnel transform) given in Eq. (ㅁ) [1]:

$$
\begin{aligned}
A_{r}\left(x, y, d_{r}\right)= & -\frac{i}{\lambda d_{r}} \exp \left(\frac{2 i \pi d_{r}}{\lambda}\right) \exp \left[\frac{i \pi}{\lambda d_{r}}\left(x^{2}+y^{2}\right)\right] \\
& \times \sum_{l=-L / 2}^{l=+L / 2} \sum_{k=-K / 2}^{k=+K / 2} H\left(l p_{x}, k p_{y}\right) \\
& \times \exp \left[\frac{i \pi}{\lambda d_{r}}\left(l^{2} p_{x}^{2}+k^{2} p_{y}^{2}\right)\right] \\
& \times \exp \left[-\frac{2 i \pi}{\lambda d_{r}}\left(l p_{x} x+k p_{y} y\right)\right] .
\end{aligned}
$$

The algorithm uses $(K, L)$ data points, with generally $(K, L) \geq(M, N)$. The pixel pitches of the reconstructed image in the $x$ and $y$ directions are given, respectively, by $\Delta \eta=\lambda d_{r} / L_{p_{x}}$ and $\Delta \eta=\lambda d_{r} / K_{p_{y}}$. The spatial resolution in the reconstructed plane are $\rho_{x}^{\prime}=\lambda d_{r} / N_{p_{x}}$ and $\rho_{y}^{\prime}=\lambda d_{r} / M_{p_{y}}$, respectively, for the $x-y$ directions of the set of reference axis attached to the object. The physical spatial resolutions are then given in the object plane by $\rho_{x}=\rho_{x}^{\prime} / \gamma_{\text {opt }}$ and $\rho_{y}=\rho_{y}^{\prime} / \gamma_{\mathrm{opt}}$. From the numerical computation given by Eq. (5), the amplitude and phase of the virtual field can be evaluated. The amplitude image is related to the image of the virtual object given by the lens, whereas the phase is useful to investigate refractive index variations, convection currents, or thermal gradients, occurring inside the object. For this, one has to evaluate the temporal phase differences at different instants. The optical phase when lightening is off is subtracted from that estimated at any instant of the recording. A quantitative measurement can be obtained after unwrapping the phase differences. Since the refractive index variations are encoded in the unwrapped phase, the use of the Gladstone-Dale relation leads to determining density variations.

\section{Proof of Principle}

We are interested in applying the proposed method to the visualization and analysis of a small air jet inside a molten glass at high temperature during its manufacturing process. In this paper, we present a proof of principle that was obtained with a light bulb submitted to a current to produce light. Holograms

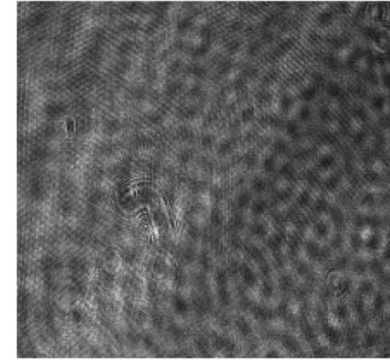

(a)

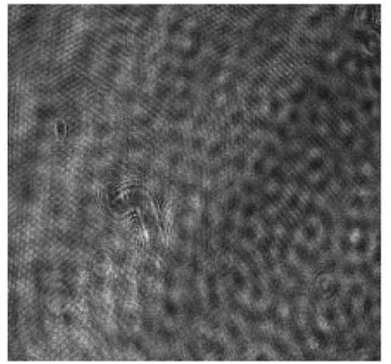

(b) (c)

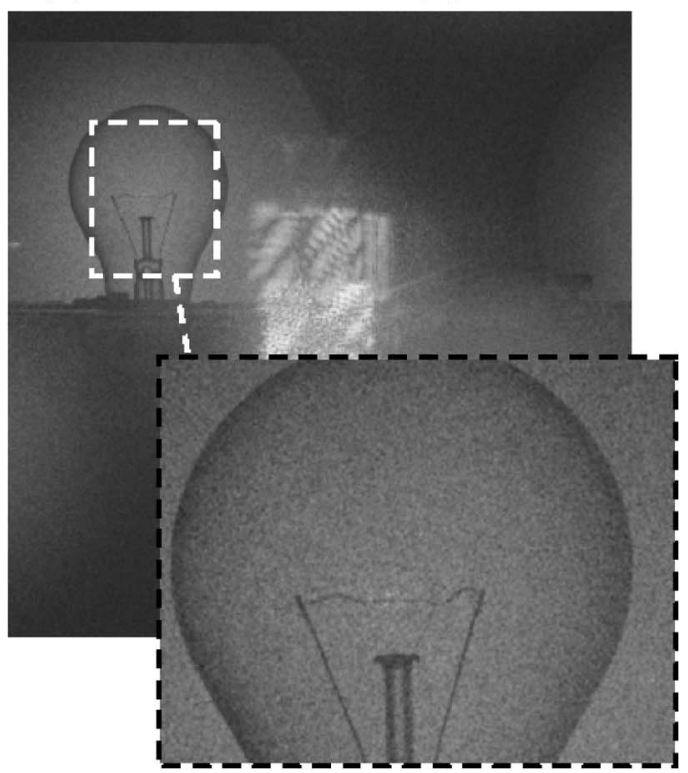

Fig. 5. Experimental results. (a) Recorded stochastic digital hologram without lightening the lamp, (b) recorded stochastic digital hologram with lightening, and (c) full-field reconstructed image amplitude obtained with the discrete Fresnel transform.

were recorded at different instants after the bulb lightening. In this paper, the diffuser illuminating the object is sized $10 \mathrm{~cm} \times 20 \mathrm{~cm}$ and a superposition tolerance of $\alpha=20 \%$ is accepted. For a physical distance $d_{0}=1150 \mathrm{~mm}$, the evaluation of the virtual distance and the focal length leads to, respectively, $d_{0}^{\prime}=275 \mathrm{~mm}$ and $f^{\prime}=-200 \mathrm{~mm}$. The optical magnification provided by the negative lens is then about $\gamma_{\text {opt }}=0.166$. The digital holograms are reconstructed with $K=L=2048$ data points, and $d_{r}=-d_{0}^{\prime}=$ $-275 \mathrm{~mm}$.

Figure $\underline{5}$ shows experimental results. Figure $\underline{5(a)}$ shows the stochastic recorded hologram when the bulb is off and Fig. 3(b) shows that recorded when the bulb is lightening. Note that the speckle nature of the hologram is clearly observable, as for Fig. 2(a) (left). Figure 5(c) shows the amplitude image obtained with the discrete Fresnel transform. The stochastic screen and the bulb can be clearly seen in the upper left corner of the field of view. Note also the slight superposition of the useful +1 order with the 0 order $(\alpha \approx 0.2)$, in the lower right part of the image of the screen. The strand of the bulb can be clearly seen. Since the spatial resolutions in the virtual plane are $\rho_{x}^{\prime}=20.9 \mu \mathrm{m}$ and $\rho_{y}^{\prime}=27.8 \mu \mathrm{m}$, 
the spatial resolutions in the physical image plane are estimated at about $\rho_{x}=125 \mu \mathrm{m}$ and $\rho_{y}=167 \mu \mathrm{m}$.

Note that the lightening of the lamp may dazzle the sensor during the recording of the holograms. To avoid this problem, a specific filter centered on the laser wavelength $(532 \mathrm{~nm})$ with a spectral bandwidth of $10 \mathrm{~nm}$ was located in front of the camera.

Figure $\underline{6}$ shows quantitative phase measurements obtained with two consecutively recorded holograms. Figures 6(a) and 6(b) show, respectively, the modulo $2 \pi$ optical phase without and with lightening of the bulb. The random nature of the phase, induced by the stochastic recording, can be clearly seen. The phase difference is given in Fig. 6(c) and shows modulo $2 \pi$ digital fringes. These "numerical fringes" exhibit the refractive index variations integrated along the optical path in the air and the glass envelope of the bulb. Figure 6(d) shows the unwrapped phase map obtained from the results in Fig. 6(c). One can note a very large amplitude variation since the phase values are in the range 10-50 radians [see the colorbar in Fig. 6(d)]. This measurement includes the contribution due to the refractive index change in the bulb and also a contribution due to the dilatation of the envelope and its refractive index variation due to the temperate increase inside the lamp $\left(\approx 500^{\circ} \mathrm{C}\right)$. For a Pyrex glass (dilatation coefficient at $3.3 \times 10^{-6}$, refractive index at 1.470 ) with thickness at $0.2 \mathrm{~mm}$, the contribution due to both thermal dilatation and refractive index change is estimated at $-3.75 \mathrm{rad}$. This means that the main part of the amplitude of the measured phase is related to the air density variations inside the bulb

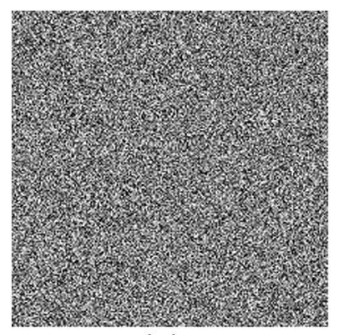

(a)

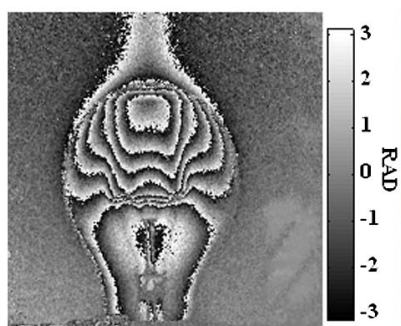

(c)

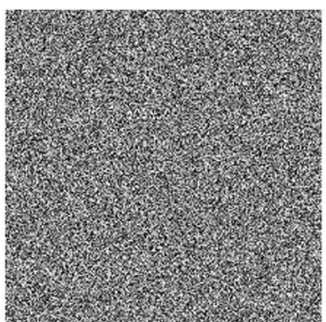

(b)

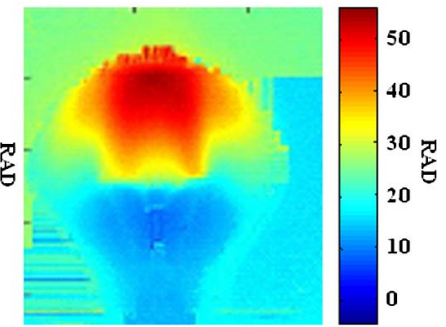

(d)
Fig. 6. Quantitative measurement inside the object, (a) virtual phase extracted from numerical reconstruction without lightening; (b) virtual phase extracted from numerical reconstruction with lightening; (c) modulo $2 \pi$ phase change calculated from (a) and (b); and (d) unwrapping of (c).

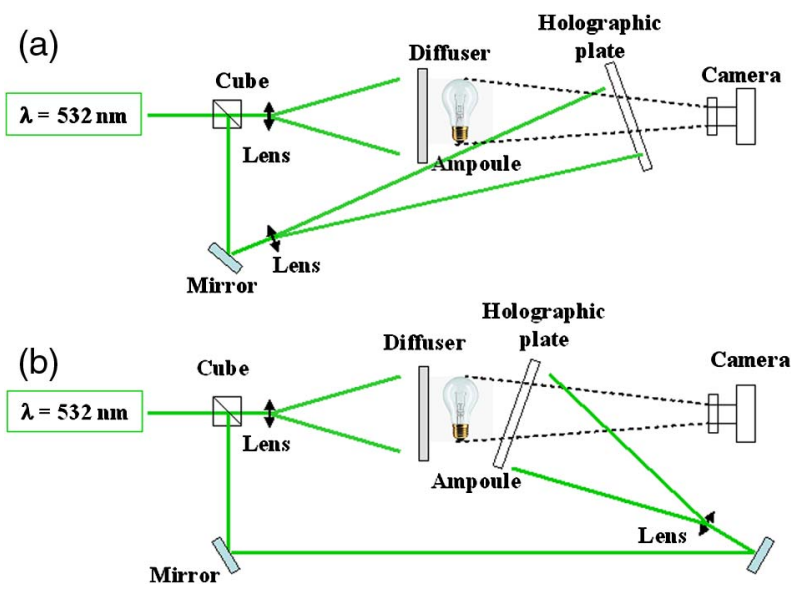

Fig. 7. Setups for silver-halide real-time holographic interferometry, (a) transmission holographic interferometer and (b) reflection holographic interferometer.

(influence of convection currents and thermal gradients).

\section{Comparison with Silver-Halide Plate Holographic Interferometry}

In order to validate the experimental results obtained with the proposed method, results obtained with silver-halide real-time holographic interferometry were compared [61-63]. To do this, two different optical setups were implemented: the first one uses transmission holographic plate as the reference wave, whereas the second one uses reflection holographic plate as the reference wave. Both setups are described in Fig. 7. Figure 7(a) shows the transmission holography mode, and Fig. 7(b) shows that for reflection holography. Note that silver-halide holographic plates were used and that the size of silver grains is about $8 \mathrm{~nm}$ [64]. Such materials provide very high quality holograms.

In the setup of Fig. 7(a), the object and reference waves are impacting the plate on the same side. In this case, the interference fringes are recorded perpendicularly to the plate surface, and the diffraction efficiency reaches only $10 \%-15 \%$. The interference fringes are not very well contrasted, but the implementation is very simple as it is easy to put the holographic plate at the same place as that it occupied during the recording. Photography of the setup is shown in Fig. 8. In the setup of Fig. 7(b), the object and reference waves are impacting the plate on both sides. Now, the interference fringes are recorded parallel to the plate surface. The diffraction efficiency can reach $100 \%$. In both setups, the image sensor includes a lens assembly to produce an image of the lamp in the pixel plane. To avoid dazzle of the sensor during the recording, a diaphragm is inserted in the lens assembly, in front of the sensor.

The sensor in Fig. 7 has the same characteristics as that used in Fig. $\underline{3}$ to record the digital holograms.

In both setups, a diffusing screen located in the front of the lamp is also used to get a stochastic 


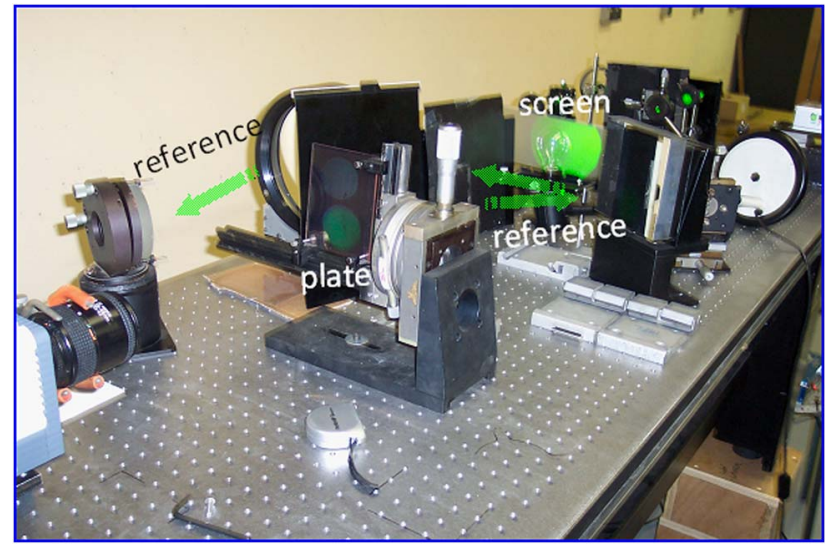

Fig. 8. Photograph of the transmission holographic interferometer.

illumination of the object. The process to get holograms of the lamp is as follows: record a transmission or reflection hologram, apply the chemical treatment to the plate to develop, bleach, dry the plate, and put the holographic plate in the setup anew (exactly at the same location as during the recording). At this step the holographic image of the bulb is observable when the reference wave is illuminating the holographic plate. Thus, it constitutes a reference object wave. Then, the lens assembly is adjusted to produce an in-focus image in the sensor plane. When illuminating both the holographic plate and the diffusing screen, real-time interferences between the reference bulb and that currently submitted to the current can be recorded by the sensor. Note that only the interference fringes can be recorded, and not the optical phase of each individual wave, whereas the digital holographic method does record the complex field.

Figure 9 shows a comparison between results obtained with stochastic digital holography and those obtained with silver-halide holographic interferometry. In both cases, recordings are performed about $20 \mathrm{~s}$ after the lightening of the lamp. The air density gradients and the convection are then well stabilized. Figure 9(a) shows the interference image obtained with the amplitude and phase change measured by stochastic digital holography. To do this, the intensity is calculated according to Eq. ( $\underline{6})$ :

$$
I=A_{r}^{\prime}(1+\cos (\Delta \varphi)),
$$

where $\Delta \varphi$ is the phase change between the current instant and the instant when lightening is off, and $A_{r}^{\prime}$ is the amplitude of the reconstructed image given by the discrete Fresnel transform. Figure 9(b) shows the interference fringes obtained with the setup of Fig. 7(a), and Fig. 9(c) shows those obtained with

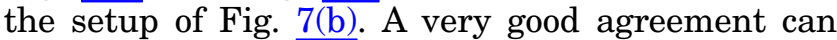
be observed between the three experimental results. Furthermore, the image quality given by each method can be appreciated. The images given by silver-halide holographic interferometry include the best spatial resolution. The strand of the lamp
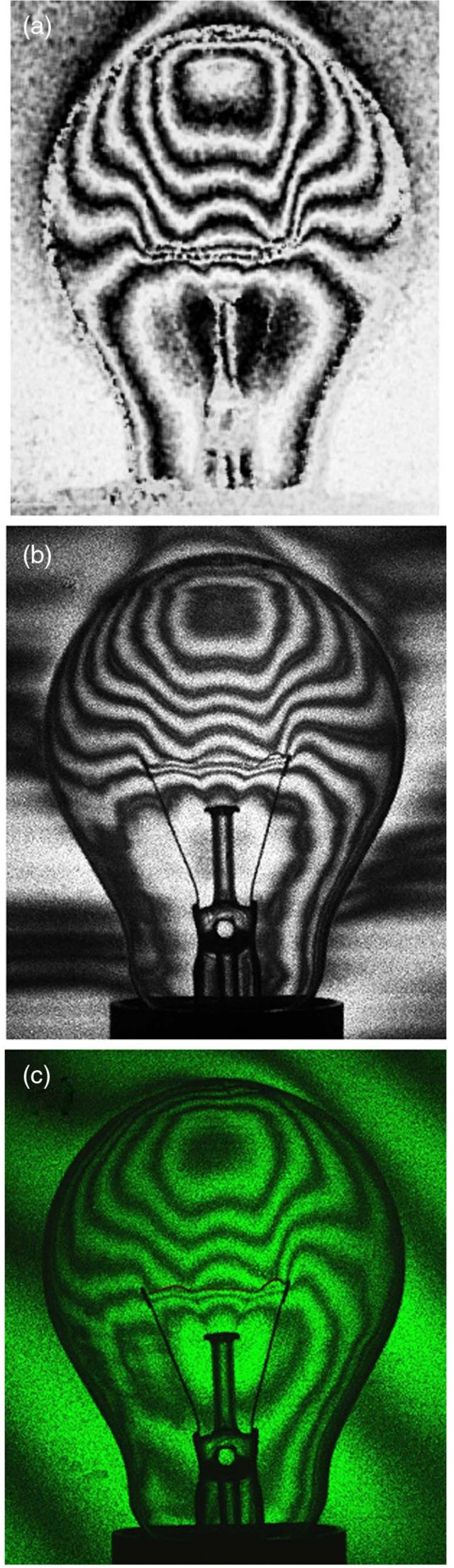

Fig. 9. Comparison between intensity of fringes, (a) fringes calculated with stochastic digital holography, (b) fringes obtained with transmission holography, and (c) fringes obtained with reflection holography. 
can be clearly seen in Figs. 6(a)-6(c). However, digital holography is more flexible since no chemical processing is required and the optical phase can be obtained from each individual hologram.

In holographic interferometry, with transmission or reflection modes, the spatial resolution of the diffracted image by the hologram owns a very high spatial resolution ( 10 or 12 thousand lines per $\mathrm{mm}$ due to the very small size silver-halide grains). So, the image resolution is determined by the sensor resolution. With a pixel pitch at $3.65 \mu \mathrm{m}$, the equivalent pitch in the image plane is estimated at $\approx 73$ microns, since the optical magnification provided by the lens assembly is $\approx 1 / 20$. Thus, the images recorded with the silver-halide holography have a slightly better resolution than that obtained with stochastic digital holography, the ratio being, respectively, 1.64 and 2.28 in the $x$ and $y$ directions.

That is an interesting feature of the digital holographic approach. The very good quality of the reconstructed images can also be perceived with the zoom in Fig. $\underline{5}$, the strand of the lamp being correctly reconstructed.

\section{Conclusion}

As a conclusion, this paper proposes a digital holographic method to visualize and measure refractive index variations, convection currents, or thermal gradients, occurring inside a transparent but strongly refracting object. The proof of principle is provided through the visualization of air density variations inside a lighting bulb. Comparisons with transmission and reflection holography demonstrate the high image and phase quality that can be extracted from the stochastic digital holographic setup.

\section{References}

1. U. Schnars and W. Jüptner, "Direct recording of holograms by a CCD target, and numerical reconstruction," Appl. Opt. 33, 179-181 (1994).

2. Th. Kreis, M. Adams, and W. Jüptner, "Methods of digital holography: a comparison,” Proc. SPIE 3098, 224-233 (1997).

3. I. Yamaguchi and T. Zhang, "Phase-shifting digital holography," Opt. Lett. 22, 1268-1270 (1997).

4. E. Cuche, F. Bevilacqua, and C. Depeursinge, "Digital holography for quantitative phase-contrast imaging," Opt. Lett. 24, 291-293 (1999).

5. C. Mann, L. Yu, C.-M. Lo, and M. Kim, "High-resolution quantitative phase-contrast microscopy by digital holography," Opt. Express 13, 8693-8698 (2005).

6. B. Bhaduri, H. Pham, M. Mir, and G. Popescu, "Diffraction phase microscopy with white light," Opt. Lett. 37, 10941096 (2012).

7. P. Girshovitz and N. T. Shaked, "Compact and portable low-coherence interferometer with off-axis geometry for quantitative phase microscopy and nanoscopy," Opt. Express 21, 5701-5714 (2013).

8. G. Rajshekhar, B. Bhaduri, C. Edwards, R. Zhou, L. L. Goddard, and G. Popescu, "Nanoscale topography and spatial light modulator characterization using wide-field quantitative phase imaging," Opt. Express 22, 3432-3438 (2014).

9. P. Ferraro, D. Alferi, S. De Nicola, L. De Petrocellis, A. Finizio, and G. Pierattini, "Quantitative phase-contrast microscopy by a lateral shear approach to digital holographic image reconstruction," Opt. Lett. 31, 1405-1407 (2006).

10. E. Shaffer, C. Moratal, P. Magistretti, P. Marquet, and C. Depeursinge, "Label-free second-harmonic phase imaging of biological specimen by digital holographic microscopy," Opt. Lett. 35, 4102-4104 (2010).

11. H. Gabai and N. T. Shaked, "Dual-channel low-coherence interferometry and its application to quantitative phase imaging of fingerprints," Opt. Express 20, 26906-26912 (2012).

12. A. B. Parthasarathy, K. K. Chu, T. N. Ford, and J. Mertz, "Quantitative phase imaging using a partitioned detection aperture," Opt. Lett. 37, 4062-4064 (2012).

13. C. Mann, P. R. Bingham, V. C. Paquit, and K. W. Tobin, "Quantitative phase imaging by three-wavelength digital holography," Opt. Express 16, 9753-9764 (2008).

14. P. Picart and M. Malek, "Complex field recovering from in-line digital holography," Opt. Lett. 38, 3230-3232 (2013).

15. S. Seebacher, W. Osten, T. Baumbach, and W. Juptner, "The determination of material parameters of microcomponents using digital holography," Opt. Lasers Eng. 36, 103-126 (2001).

16. I. Yamaguchi, T. Ida, M. Yokota, and K. Yamashita, "Surface shape measurement by phase shifting digital holography with a wavelength shift," Appl. Opt. 45, 7610-7616 (2006).

17. T. Nomura, B. Javidi, S. Murata, E. Nitanai, and T. Numata, "Polarization imaging of a 3D object by use of on-axis phaseshifting digital holography," Opt. Lett. 32, 481-483 (2007).

18. Y. Morimoto, T. Nomura, M. Fjigaki, S. Yoneyama, and I. Takahashi, "Deformation measurement by phase shifting digital holography," Exp. Mech. 45, 65-70 (2005).

19. P. Tankam, Q. Song, M. Karray, J. Li, J.-M. Desse, and P. Picart, "Real-time three-sensitivity measurements based on three-color digital Fresnel holographic interferometry," Opt. Lett. 35, 2055-2057 (2010).

20. Y. Fu, G. Pedrini, and W. Osten, "Vibration measurement by temporal Fourier analyses of a digital hologram sequence," Appl. Opt. 46, 5719-5727 (2007).

21. M. Leclercq, M. Karray, V. Isnard, F. Gautier, and P. Picart, "Evaluation of surface acoustic waves on the human skin using quasi-time-averaged digital Fresnel holograms," Appl. Opt. 52, A136-A146 (2013).

22. F. Joud, F. Verpillat, F. Laloë, M. Atlan, J. Hare, and M. Gross, "Fringe-free holographic measurements of large-amplitude vibrations," Opt. Lett. 34, 3698-3700 (2009).

23. B. Samson, F. Verpillat, M. Gross, and M. Atlan, "Video-rate laser Doppler vibrometry by heterodyne holography," Opt. Lett. 36, 1449-1451 (2011).

24. I. Yamaguchi, T. Matsumura, and J. Kato, "Phase shifting color digital holography," Opt. Lett. 27, 1108-1110 (2002).

25. J. Zhao, H. Jiang, and J. Di, "Recording and reconstruction of a color holographic image by using digital lensless Fourier transform holography," Opt. Express 16, 2514-2519 (2008).

26. T. Kakue, T. Tahara, K. Ito, Y. Shimozato, Y. Awatsuji, K. Nishio, S. Ura, T. Kubota, and O. Matoba, "Parallel phaseshifting color digital holography using two phase shifts," Appl. Opt. 48, H244-H250 (2009).

27. T. Kakue, K. Ito, Y. Awatsuji, K. Nishio, T. Kubota, and O. Maoba, "Parallel phase-shifting digital holography capable of simultaneously capturing visible and invisible threedimensional information," J. Disp. Technol. 6, 472-478 (2010).

28. J. Garcia-Sucerquia, "Color lensless digital holographic microscopy with micrometer resolution," Opt. Lett. 37, 1724-1726 (2012).

29. N. Demoli, D. Vukicevic, and M. Torzynski, "Dynamic digital holographic interferometry with three wavelengths," Opt. Express 11, 767-774 (2003).

30. P. Tankam and P. Picart, "Use of digital color holography for crack investigation in electronic components," Opt. Lasers Eng. 49, 1335-1342 (2011).

31. G. Pedrini, P. Fröning, H. J. Tiziani, and M. E. Gusev, "Pulsed digital holography for high-speed contouring that uses a two-wavelength method," Appl. Opt. 38, 3460-3467 (1999).

32. C. Wagner, W. Osten, and S. Seebacher, "Direct shape measurement by digital wave front reconstruction and multi-wavelength contouring," Opt. Eng. 39, 79-85 (2000).

33. A. Wada, M. Kato, and Y. Ishii, "Multiple-wavelength digital holographic interferometry using tunable laser diodes," Appl. Opt. 47, 2053-2060 (2008).

34. J. Kuhn, T. Colomb, F. Montfort, F. Charriere, Y. Emery, E. Cuche, P. Marquet, and C. Depeursinge, "Real-time 
dual-wavelength digital holographic microscopy with a single hologram acquisition," Opt. Express 15, 7231-7242 (2007).

35. A. Wada, M. Kato, and Y. Ishii, "Large step-height measurements using multiple-wavelength holographic interferometry with tunable laser diodes," J. Opt. Soc. Am. A 25, 3013-3020 (2008).

36. C. J. Mann, P. R. Bingham, V. C. Paquit, and K. W. Tobin, "Quantitative phase imaging by three-wavelength digital holography," Opt. Express 16, 9753-9764 (2008).

37. P. Picart, P. Tankam, D. Mounier, Z. Peng, and J. Li, "Spatial bandwidth extended reconstruction for digital color Fresnel holograms," Opt. Express 17, 9145-9156 (2009).

38. P. Xia, Y. Shimozato, Y. Ito, T. Tahara, T. Kakue, Y. Awatsuji, K. Nishio, S. Ura, T. Kubota, and O. Matoba, "Improvement of color reproduction in color digital holography by using spectral estimation technique," Appl. Opt. 50, H177-H182 (2011).

39. Y. Ito, Y. Shimozato, P. Xia, T. Tahara, T. Kakue, Y. Awatsuji, K. Nishio, S. Ura, T. Kubota, and O. Matoba, "Fourwavelength color digital holography," J. Disp. Technol. 8, 570-576 (2012).

40. A. Kowalczyk, M. Bieda, M. Makowski, M. Sypek, and A. Kolodziejczyk, "Fiber-based real-time color digital in-line holography," Appl. Opt. 52, 4743-4748 (2013).

41. M. Kim, "Full color natural light holographic camera," Opt. Express 21, 9636-9642 (2013).

42. M. Paturzo, P. Ferraro, S. Grilli, D. Alfieri, P. De Natale, M. de Angelis, A. Finizio, S. De Nicola, G. Pierattini, F. Caccavale, D. Callejo, and A. Morbiato, "On the origin of internal field in lithium niobate crystals directly observed by digital holography," Opt. Express 13, 5416-5423 (2005).

43. P. Picart, J. Leval, F. Piquet, J. Boileau, T. Guimezanes, and J. P. Dalmont, "Tracking high amplitude auto-oscillations with digital Fresnel holograms," Opt. Express 15, 8263-8274 (2007).

44. N. Warnasooriya, F. Joud, P. Bun, G. Tessier, M. Coppey-Moisan, P. Desbiolles, M. Atlan, M. Abboud, and M. Gross, "Imaging gold nanoparticles in living cell environments using heterodyne digital holographic microscopy," Opt. Express 18, 3264-3273 (2010).

45. N. Verrier, D. Alexandre, and M. Gross, "Laser Doppler holographic microscopy in transmission: application to fish embryo imaging," Opt. Express 22, 9368-9379 (2014).

46. M. Locatelli, E. Pugliese, M. Paturzo, V. Bianco, A. Finizio, A. Pelagotti, P. Poggi, L. Miccio, R. Meucci, and P. Ferraro, "Imaging live humans through smoke and flames using farinfrared digital holography," Opt. Express 21, 5379-5390 (2013).

47. J. M. Desse, P. Picart, and P. Tankam, "Digital three-color holographic interferometry for flow analysis," Opt. Express 16, 5471-5480 (2008).
48. T. Kakue, R. Yonesaka, T. Tahara, Y. Awatsuji, K. Nishio, S. Ura, T. Kubota, and O. Matoba, "High-speed phase imaging by parallel phase-shifting digital holography," Opt. Lett. 36, 4131-4133 (2011).

49. J. M. Desse, P. Picart, and P. Tankam, "Digital color holography applied to fluid and structural mechanics," Opt. Lasers Eng. 50, 18-28 (2012).

50. R. Dolelek, P. Psota, V. Lédl, T. Vít, J. Václavík, and V. Kopecký, "General temperature field measurement by digital holography," Appl. Opt. 52, A319-A325 (2013).

51. V. Kumar, M. Kumar, and C. Shakher, "Measurement of natural convective heat transfer coefficient along the surface of a heated wire using digital holographic interferometry," Appl. Opt. 53, G74-G83 (2014).

52. M. Hossain and C. Shakher, "Temperature measurement in laminar free convective flow using digital holography," Appl. Opt. 48, 1869-1877 (2009).

53. S. Sharma, G. Sheoran, and C. Shakher, "Temperature measurement of axisymmetric flame under the influence of magnetic field using lensless Fourier transform digital holography," Appl. Opt. 51, 4554-4562 (2012).

54. S. Sharma, G. Sheoran, and C. Shakher, "Digital holographic interferometry for measurement of temperature in axisymmetric flames," Appl. Opt. 51, 3228-3235 (2012).

55. N. Demoli, J. Mestrovic, and I. Sovic, "Subtraction digital holography," Appl. Opt. 42, 798-804 (2003).

56. P. Picart and J. Leval, "General theoretical formulation of image formation in digital Fresnel holography," J. Opt. Soc. Am. A 25, 1744-1761 (2008).

57. U. Schnars, T. M. Kreis, and W. O. Jüptner, "Digital recording and numerical reconstruction of holograms: reduction of the spatial frequency spectrum," Opt. Eng. 35, 977-982 (1996).

58. J. Mundt and T. Kreis, "Digital holographic recording and reconstruction of large scale objects for metrology and display," Opt. Eng. 49, 125801 (2010).

59. J. W. Goodman, Introduction to Fourier Optics, 2nd ed. (McGraw-Hill, 1996).

60. P. Picart and P. Tankam, "Analysis and adaptation of convolution algorithms to reconstruct extended objects in digital holography," Appl. Opt. 52, A240-A253 (2013).

61. J. M. Desse and J. L. Tribillon, "Real-time three-color reflection holographic interferometer," Appl. Opt. 48, 6870-6877 (2009).

62. J. M. Desse, F. Albe, and J. L. Tribillon, "Real-time color holographic interferometry," Appl. Opt. 41, 5326-5333 (2002).

63. I. Naydenova, Advanced Holography-Metrology and Imaging (InTech open, 2011)

64. Y. Gentet, The Ultimate Holography, http://www.ultimateholography.com/. 